\title{
JACOB KOREVAAR
}

\section{Electrostatic fields due to distributions of electrons}

Annales de la faculté des sciences de Toulouse $6^{e}$ série, tome S5 (1996), p. 57-76

<http://www.numdam.org/item?id=AFST_1996_6_S5_57_0>

(C) Université Paul Sabatier, 1996, tous droits réservés.

L'accès aux archives de la revue «Annales de la faculté des sciences de Toulouse » (http://picard.ups-tlse.fr/ annales/) implique l'accord avec les conditions générales d'utilisation (http://www.numdam.org/conditions). Toute utilisation commerciale ou impression systématique est constitutive d'une infraction pénale. Toute copie ou impression de ce fichier doit contenir la présente mention de copyright.

\section{NumDam}

Article numérisé dans le cadre du programme Numérisation de documents anciens mathématiques http://www.numdam.org/ 


\title{
Electrostatic fields due to distributions of electrons
}

\author{
JACOB KorevaAR ${ }^{(1)}$
}

\section{Introduction}

Working in $\mathbb{R}^{s}(s \geq 2)$ with the classical potential, we let $K$ denote a compact set ("conductor") of positive capacity. By $\omega$ we denote the unique distribution of positive charge on $K$ of total charge 1 with minimal potential energy. This probability measure defines the so-called classical equilibrium distribution for $K$, whose support belongs to the outer boundary $\partial_{0} K$, cf. Frostman [5]. We wish to approximate $\omega$ by distributions (probability measures) $\mu_{N}=\mu_{N}\left(x_{1}, \ldots, x_{N}\right)$ which consist of $N$ point masses or charges $1 / N$ ("electrons") at points $x_{1}, \ldots, x_{N}$ of $K$. If one imposes the condition of minimal potential energy on such discrete measures, one obtains what we call a "Fekete equilibrium distribution" $\omega_{N}$. Its support is a set of $N$ th order "Fekete points" $f_{N 1}, \ldots, f_{N N}$ for $K$, which are located on $\partial_{0} K$. For planar sets, such points were introduced by Fekete in 1923 [4]. Instead of minimizing the energy, he (equivalently) maximized the product

$$
\prod_{1 \leq j<k \leq N}\left|x_{j}-x_{k}\right|^{2}, \quad x_{1}, \ldots, x_{N} \in K
$$

The quality of the approximation to $\omega$ by $\mu_{N}$ can be measured in different ways. One may estimate the differences $\omega(E)-\mu_{N}(E)$ for suitably regular sets $E$, or one may compare the potentials $U^{\omega}$ and $U^{\mu_{N}}$ or the electrostatic fields $\mathcal{E}^{\omega}$ and $\mathcal{E}^{\mu_{N}}$. The paper surveys results on the following main problems.

(1) Department of Mathematics, University of Amsterdam, Plantage Muidergracht 24, 1018 TV Amsterdam (Netherlands) 
Problem 1. - It should be of physical interest to compare the classical equilibrium field with "Fekete fields" for large $N$. How small are the differences $\mathcal{E}^{\omega}-\mathcal{E}^{\omega_{N}}$ away from the outer boundary $\partial_{0} K$ ?

One knows that $\mathcal{E}^{\omega}=0$ throughout the interior of the conductor $K$ (Faraday cage phenomenon of electrostatics). Question: How small is $\mathcal{E}^{\omega_{N}}$ inside $K$ for large $N$ ? In other words, how well can one explain the Faraday cage phenomenon on the basis of a model, in which the total charge 1 on the conductor is made up of a large number of equal point charges?

Problem 2.- How small can one make $\mathcal{E}^{\omega}-\mathcal{E}^{\mu_{N}}$ for large $N$ if one allows arbitrary distributions $\mu_{N}$ of $N$ point charges $1 / N$ on $\partial_{0} K$ ?

Beginning in 1885, STIELTJEs devoted a number of papers to equilibrium problems for point charges on a segment in the plane and related matters, see [29]-[32] and cf. Van Assche [34]. Among other things STIELTJEs' work contains a description of the Fekete points for the case where $K$ is the interval $[-1,1]$, cf. the Examples 2.1 below.

For a conductor $K$ given by an analytic or $C^{3, \alpha}$ Jordan curve $\Gamma$ in the plane, Pommerenke ([24], [25]) and Korevaar and Kortram ([11], [15]) have obtained close approximations to the Fekete points. For very smooth $\Gamma$, the approximations show that $\omega(E)-\omega_{N}(E)=\mathcal{O}(1 / N)$, uniformly for the subarcs $E \subset \Gamma$. Via a Stieltues integral for the potential difference $U^{\omega}-U^{\omega_{N}}$, the approximations imply that $\mathcal{E}^{\omega}-\mathcal{E}^{\omega_{N}}$ is at most of order $1 / N$ at distance $\geq \epsilon>0$ from the curve, see horevaar and coauthors Geveci and Kortram ([11], [14, [15]). This order is sharp except when $\Gamma$ is a circle.

For less smooth conductors in the plane (such as a square) and for conductors in higher dimensions, there are separation results for the points in Fekete $N$-tuples (Kövari and Pommerenke [19], [20]; Dahlberg [3]), but only the beginnings of good approximations, cf. Sjögren [28]. Without explicit information about the Fekete points, Korevaar and his student Monterie have recently obtained rather precise estimates for $\mathcal{E}^{\omega}-\mathcal{E}^{\omega_{N}}$ in the case of convex or smoothly bounded sets $K$. In $\mathbb{R}^{3}$ the basic order is $1 / \sqrt{N}$. The precise results will be stated and the various steps in the proof will be sketched in Section 3 .

On Problem 2 it is known that at least for smooth Jordan curves in the plane different from circles, the Fekete fields give much worse approximations to the classical equilibrium field than the fields due to certain other $N$-tuples of point charges $1 / N$, cf. Korevaar and Geveci [14] for the case of analytic curves. In the present paper we will restrict ourselves 
to the case of the unit sphere $S$ in $\mathbb{R}^{3}$ where the situation is unclear. For the sphere, Problem 2 is of interest because of its relation to Chebyshev-type quadrature formulas, that is, quadrature formulas in which all $N$ nodes carry the same weight. Very good $N$-tuples of Chebyshev nodes on $S$ correspond to $N$-tuples of point charges $1 / N$ on the sphere for which the electrostatic field is extremely small on compact subsets of the unit ball, see Section 4 and Korevaar and Meyers [16]. The optimal order of smallness lies between $\exp \left(-c N^{1 / 3}\right)$ and $\exp \left(-c N^{1 / 2}\right)[16]$.

It is conjectured that the latter order can be achieved. A proof may be derived from a plausible (but unproved) separation result for the $N$ th order Fekete points on the sphere relative to the nonstandard potential given by (2.4) below, see Theorem 4.3 and cf. Korevaar [13].

\section{Basic notions and results from potential theory}

Throughout the paper, $\mu$ stands for an arbitrary probability measure on the compact set $K \subset \mathbb{R}^{s}$. It is convenient to start with general (repulsive) potentials of the form

$$
U^{\mu}(x)=\int_{K} \Phi(|x-y|) \mathrm{d} \mu(y),
$$

where $\Phi(r)$ is smooth on $(0 . \infty)$ and strictly decreasing. The field and the potential energy are given by

$$
\mathcal{E}^{\mu}(x)=-\operatorname{grad} U^{\mu}(x), \quad I(\mu)=\int_{K^{-} \times K} \Phi(|x-y|) \mathrm{d} \mu(x) \mathrm{d} \mu(y) .
$$

We are primarily interested in the classical potential for $\mathbb{R}^{s}$ which corresponds to

$$
\Phi(r)= \begin{cases}1 / r^{s-2} & \text { if } s>2 \\ \log (1 / r) & \text { if } s=2\end{cases}
$$

For $\Phi(r)=1 / r^{\alpha}(0<a<s)$ one obtains the Riesz potentials. In $\mathbb{R}^{3}$ another interesting potential is given by

$$
\Phi(r)=1 / \sqrt{r^{2}+a^{2}} \quad(a>0) .
$$

Let us first assume $\inf _{\mu} I(\mu)<\infty$. For the classical potential Frostman [5] proved that there is a unique probability measure $\mu$ on $K$ with minimal 
potential energy, the "equilibrium distribution" $\omega$ for $K$ with the following properties:

$$
\begin{aligned}
I(\omega) & =V=V_{K} \stackrel{\text { def }}{=} \min I(\mu), \\
U^{\omega} & \leq V \text { throughout } \mathbb{R}^{s}, \\
U^{\omega} & =V \text { inside } K \text { and quasi everywhere on } \partial_{0} K,
\end{aligned}
$$

so that $\mathcal{E}^{\omega}=0$ inside $K$. If $\inf I(\mu)=\infty$ one sets $V=\infty$. The constant $V=V_{K}$ is called "Robin's constant" (after the mathematical physicist Robin [26]). It is customary to define

$$
\text { cap } K=\Phi^{-1}\left(V_{K^{-}}\right) \text {, }
$$

so that the capacity has the dimension of a length. The expression "quasi everywhere" above means: everywhere outside an exceptional set of (outer) capacity zero. The preceding results extend to the Riesz potentials with $s-2<\alpha<s$, cf. Frostman [5].

We return to the classical case, in which supp $\omega \subset \partial_{0} K$. If the outer boundary $\partial_{0} K$ is such that its exterior $K^{\infty}$ relative to $\mathbb{R}^{s} \cup\{\infty\}$ is regular for the Dirichlet problem for harmonic functions, there is no exceptional set. In that case one may define a Green's function $G(y, \infty)$ for $K^{\infty}$ with pole at $\infty$ as follows. Excluding the case $s=2$ for a moment, $G(y, \infty)$ is the harmonic function on $K^{-\infty}-\{\infty\}$ with boundary values 0 on $\partial K^{\infty}=\partial_{0} K$ and 1 at $\infty$. Thus for $s \geq 3$,

$$
\begin{aligned}
G(y, \infty) & =1-U^{\omega}(y) / V \quad \text { for } y \in K^{\infty} \\
& =1-\left(\frac{\operatorname{cap} K}{|y|}\right)^{s-2}+\mathcal{O}\left(\frac{1}{|y|^{s-1}}\right) \quad \text { as } y \rightarrow \infty .
\end{aligned}
$$

In the case $s=2$ one has $G(y, \infty)=V-U^{\omega}(y)$. For well-behaved outer boundary $\partial_{0} K$ one can express the equilibrium measure in terms of the normal derivative $\left(\partial G / \partial n_{y}\right)(y, \infty)$, see Theorem 6.3.

As stated in the Introduction, we also consider special discrete probability measures

$$
\mu_{N^{*}}=\mu_{N}\left(x_{1}, \ldots, x_{N}\right) \quad\left(\Lambda^{*} \geq 2\right)
$$

given by charges $1 / N$ at points $x_{1}, \ldots, x_{N} \in K$. The corresponding potential and discrete energy are

$$
U^{\mu_{N}}(x)=\frac{1}{N} \sum_{k=1}^{N} \Phi\left(\left|x-x_{k}\right|\right), I^{*}\left(\mu_{N}\right)=\frac{1}{N^{2}} \sum_{j . k=1, j \neq k}^{N} \Phi\left(\left|x_{j}-x_{k}\right|\right) .
$$

(Observe that the "ordinary" energy $I\left(\mu_{N}\right)$ may be equal to $\propto$.) 
Any $\mu_{N}$ with minimal discrete energy will be denoted by $\omega_{N}$ and called an $N$ th order Fekete measure on $K$. The carrying points are $N$ th order Fekete points $f_{N 1}, \ldots, f_{N N}$. For the classical potential they lie on $\partial_{0} K$. Fekete $N$-tuples need not be unique.

Examples 2.1.- On the unit circle or the closed unit disc in the plane, the Fekete $N$-tuples are given by the $N$ th roots of unity or their rotations through a fixed angle, cf. Schur [27].

For the segment $[-1,1]$ in the plane, STIELTJES' results of $1885-86$ imply that the Fekete points are the zeros of $\left(1-x^{2}\right) P_{N-1}^{\prime}(x)$, where $P_{k}$ denotes the Legendre polynomial of degree $k$, cf. Stieltjes [29]-[32], Schur [27], Szegő's book [33, Sect. 6.7] and Van Assche [34].

In the case of the unit sphere in $\mathbb{R}^{3}$, we will also be interested in the Fekete points for the potential given by (2.4).

It follows from Frostman's work that for the classical potentials (as well as the Riesz potentials with $s-2<\alpha<s$ ) and every compact set $K$ of positive capacity,

$$
\omega_{N} \rightarrow \omega \text { in weak* sense as } N \rightarrow \infty,
$$

cf. Frostman [5, pp. 47 and 10].

\section{Fekete fields in $\mathbb{R}^{s}$ : new results}

In the following, $\Sigma$ will be a bounded closed (hyper)surface whose complement has two components. The interior domain will be called $\Omega$, while the exterior domain - including the point at $\infty$ - is denoted by $\Omega^{\infty}$. It will be convenient to think of $K$ as $\operatorname{clos} \Omega$. We use the classical potentials here.

Theorem 3.1 (Korevaar and Monterie [17], cf. Monterie [21]). - For $\Omega \subset \mathbb{R}^{s}$ convex or $\Sigma=\partial \Omega$ of class $C^{1, \alpha}$, and at distance $\geq \epsilon>0$ from $\Sigma$, there are uniform estimates

$$
\begin{gathered}
U^{\omega}-U^{\omega_{N}}=\mathcal{O}\left(\frac{1}{N^{1 /(s-1)}}\right), \quad \mathcal{E}^{\omega}-\mathcal{E}^{\omega_{N}}=\mathcal{O}\left(\frac{1}{N^{1 /(s-1)}}\right) \quad \text { if } s \geq 3, \\
U^{\omega}-U^{\omega_{N}}=\mathcal{O}\left(\frac{\log N}{N}\right), \quad \mathcal{E}^{\omega}-\mathcal{E}^{\omega_{N}}=\mathcal{O}\left(\frac{\log N}{N}\right) \quad \text { if } s=2 \quad(N \geq 2) .
\end{gathered}
$$


Since the proof has not appeared in a journal we will present an outline here, and provide additional technical details in Sections 5 and 6. For the time being we take $s \geq 3$.

Step 1.- In order to manufacture a positive potential, we introduce a lower bound for the difference $U^{\omega_{N}}-U^{\omega}$ on $K$ or $\Sigma$. Here $U^{\omega} \equiv V$, the Robin constant for $K$. Setting

$$
\min _{x \in \Sigma}\left\{U^{\omega_{N}}(x)-U^{\omega}(x)\right\}=-\delta\left(\omega_{N}\right),
$$

it is easy to see that $\delta\left(\omega_{N}\right)>0$. Indeed, by Fubini's theorem,

$$
\int_{\Sigma}\left(U^{\omega_{N}}-U^{\omega}\right) \mathrm{d} \omega=\int_{\Sigma} U^{\omega} \mathrm{d} \omega_{N}-\int_{\Sigma} U^{\omega} \mathrm{d} \omega=V-V=0 .
$$

Hence $U^{\omega_{N}}-U^{\omega}$, which is positive near the Fekete points, must be negative somewhere on $\Sigma$ (the support of $\omega$ is all of $\Sigma$ ).

We now form the auxiliary potential

$$
\begin{gathered}
T(x) \stackrel{\text { def }}{=} U^{\omega_{N}}(x)-\left\{1-\delta\left(\omega_{N}\right) / V\right\} U^{\omega}(x)=U^{\rho_{N}}(x), \\
\rho_{N} \stackrel{\text { def }}{=} \omega_{N}-\left\{1-\delta\left(\omega_{N}\right) / V\right\} \omega, \quad \operatorname{supp} \rho_{N} \subset \Sigma .
\end{gathered}
$$

By (3.1), $T(x) \geq 0$ on $\Sigma$, hence $T(x)>0$ throughout $\mathbb{R}^{s}-\Sigma$ (minimum principle for harmonic functions).

Step 2. - If $\Sigma$ is the unit sphere, $V=1 / \operatorname{cap} \Sigma=1$, so that $T(0)=$ $\delta\left(\omega_{N}\right)$. One may then use the Poisson integral or Harnack's inequality to prove that $T$, and hence $U^{\omega}{ }_{N}-U^{\omega}<T$, is $\leq \mathcal{O}\left(\delta\left(\omega_{N}\right)\right)$ away from the surface, cf. Korevaar [12] for $s=3$.

In the general case there is usually no finite center where one has information about the value of $T$. However, we do have information at infinity:

$$
L(T) \stackrel{\text { def }}{=} \lim _{x \rightarrow \infty}|x|^{s-2} T(x)=\delta\left(\omega_{N}\right) / V,
$$

since $\lim |x|^{s-2} U^{\mu}(x)=1$ for all probability measures $\mu$.

For $x$ in the exterior domain $\Omega^{\infty}$ one may now use a Harnack-type inequality around $\infty$ to conclude that for the positive harmonic function $T$,

$$
|x|^{s-2} T(x) \leq c\left(d_{x}\right) L(T), \quad d_{x}=d(x, \Sigma) .
$$


To verify this one may apply Kelvin inversion with center $0 \in \Omega$; for finite domains there is a general Harnack inequality in Gilbarg and Trudinger [8]. In our case, $c\left(d_{x}\right)$ turns out to be $\leq c_{1}\left(1+1 / d_{x}^{s-1}\right)$, cf. Korevaar and Monterie [17] for $s=3$.

Step 3. - We next take $x$ in the interior domain $\Omega$. For our kind of domains, there are integrals for $T(x)$ and $L(T)$ over $\Sigma$ in terms of normal derivatives of Green's functions, as well as certain useful inequalities for those derivatives, see Section 6. Using suitably normalized area measure $\sigma$, these tools enable us to estimate as follows:

$$
\begin{aligned}
T(x) & =\int_{\Sigma} T(y) \frac{\partial G}{\partial n_{y}}(y, x) \mathrm{d} \sigma(y) \leq M\left(d_{x}\right) \int_{\Sigma} T(y) \mathrm{d} \sigma(y) \\
& \leq \frac{M\left(d_{x}\right)}{m(\infty)} \int_{\Sigma} T(y) \frac{\partial G}{\partial n_{y}}(y, \infty) \mathrm{d} \sigma(y)=\frac{M\left(d_{x}\right)}{m(\infty)} L(T) \\
& \leq \frac{c_{2}}{d_{x}^{s-1}} L(T) .
\end{aligned}
$$

Indeed, $\left(\partial G / \partial n_{y}\right)(y, x)$ will be bounded above by a constant $M\left(d_{x}\right) \leq$ $c / d_{x}^{s-1}$ for $x \in \Omega$ and $y \in \Sigma$, while $\left(\partial G / \partial n_{y}\right)(y, \infty)$ is bounded from below by a positive constant $m(\infty)$ (Section 6$)$.

Step 4.- Comparison of $U^{\omega_{N}}-U^{\omega}$ on $K$ or $\Sigma$ with a difference of energies will show that under the hypotheses of Theorem 3.1,

$$
\delta\left(\omega_{N}\right) \leq \frac{c_{3}}{N^{1 /(s-1)}},
$$

see Section 5.

Step 5. - The end result from (3.1)-(3.6) is that for $N \geq 2$ and all $x \notin \Sigma$,

$$
-\frac{c_{3}}{N^{1 /(s-1)}}<U^{\omega_{N}}(x)-U^{\omega}(x)<T(x)<\frac{c_{4}\left(1+d_{x}\right)}{d_{x}^{s-1}} \frac{1}{N^{1 /(s-1)}} \quad(s \geq 3)
$$

where $d_{x}=d(x, \Sigma)$ and the constants $c_{j}$ depend only on $\Omega$.

An upper bound for $\left|\mathcal{E}^{\omega}-\mathcal{E}^{\omega_{N}}\right|$ may be obtained from (3.7) with the aid of the Poisson integral. The result is that for $N \geq 2$ and all $x \notin \Sigma$,

$$
\left|\mathcal{E}^{\omega}(x)-\mathcal{E}^{\omega_{N}}(x)\right|<\frac{c_{5}\left(1+d_{x}\right)}{d_{x}^{s}} \frac{1}{N^{1 /(s-1)}} \quad(s \geq 3) .
$$




\section{Remarks}

In the case of dimension $s=2$ one would define $T(x)=U^{\omega_{N}}(x)-U^{\omega}(x)+$ $\delta\left(\omega_{N}\right)$. Here the method of Section 5 will give $\delta\left(\omega_{N}\right)=\mathcal{O}((\log N) / N)$, hence the disappointing estimate for $s=2$ in Theorem 3.1. One has the feeling that the logarithm should not be there, cf. the known result for very smooth curves! In fact, in the degenerate case of the interval $[-1,1]$, direct computation of the difference of the potentials also gives an estimate $\mathcal{O}(1 / N)$. On the other hand it may be remarked that a related older result of Pommerenke for (simply connected) exterior domains also contains a logarithm [23].

In a way it is encouraging that the present method gives a result for smooth curves which is close to best possible. Thus the following conjecture appears to be reasonable.

CONJECTURE 3.2. - The order $1 / N^{1 /(s-1)}$ for dimensions $s \geq 3$ in Theorem 3.1 is sharp except possibly for the case where $\Sigma$ is a sphere.

\section{Small fields due to electrons on the sphere}

For the unit circle $C=C(0,1)$ in the plane, the Fekete $N$-tuples are given by the $N$ th roots of unity or their rotations through a fixed angle. Here $\left|\mathcal{E}^{\omega}(x)-\mathcal{E}^{\omega_{N}}(x)\right|$ will become exponentially small outside a fixed neighborhood of $C$ as $N \rightarrow \infty$. Restricting oneself to the interior of $C$. one finds that

$$
\sup _{|x| \leq r}\left|\mathcal{E}^{\omega_{N}}(x)\right|=\frac{r^{N-1}}{1-r^{N}} \quad \text { for } 0<r<1 .
$$

For smooth Jordan curves other than circles, very small electrostatic fields $\mathcal{E}^{\mu_{N}}$ are associated with charges at the images of $N$ th roots of unity under an exterior conformal map rather than Fekete points, cf. Korevaar and Geveci [14]. In a sense this is true even for the outer boundary of an arbitrary connected compact set, see horevaar [10].

The unit sphere $S=S(0,1) \subset \mathbb{R}^{3}$

Preliminary numerical results of Kuijlaars and Voogd in Amsterdam suggest that for the sphere $S$, the Fekete fields $\mathcal{E}^{\omega_{N}}$ do not become extremely 
small on interior balls $B(0, r)$ as $N \rightarrow \infty$. But how small can one make the fields $\mathcal{E}^{\mu_{N}}$ on such balls if one allows arbitrary $N$-tuples of point charges $1 / N$ on $S ?$

It will be convenient to introduce some new notation. In the following $Z_{N}=\left(\zeta_{1}, \ldots, \zeta_{N}\right)$ stands for an $N$-tuple of points on $S$. The corresponding $N$-tuple $\mu_{N}$ of point charges $1 / N$ will be denoted by $\mu\left(Z_{N}\right)$. For the potential $U^{\mu_{N}}$ and the electrostatic field $\mathcal{E}^{\mu_{N}}$ we will write $U\left(\cdot, Z_{N}\right)$ and $\mathcal{E}\left(\cdot, Z_{N}\right)$.

Theorem 4.1 (Korevaar and Meyers [16]). - On the unit sphere $S$ there are special $N$-tuples of points $Z_{N}$ such that for $N \rightarrow \infty$, the differences

$$
\mathcal{E}^{\omega}(x)-\mathcal{E}\left(x, Z_{N}\right)
$$

become as small as $\mathcal{O}\left(e^{-c N^{1 / 3}}\right)$ outside any given neighborhood of $S$ (with $c>0$ depending on the neighborhood). However, the differences can not become of smaller order than $e^{-c N^{1 / 2}}$.

The proof depends on the close relation between the electrostatic field $\mathcal{E}\left(\cdot, Z_{N}\right)$ inside $S$ and Chebyshev-type quadrature on $S$ with nodes $Z_{N}$ mentioned in the Introduction. Good $N$-tuples $Z_{N}$ will be described below when we discuss Chebyshev-type quadrature. As to the second part, it was shown in [16] that for every $N$-tuple $Z_{N}$ on $S$,

$$
\sup _{|x|=r}\left|\mathcal{E}\left(x, Z_{N}\right)\right|>\frac{r^{2 \sqrt{N}}}{4(\sqrt{N}+1)^{3}}, \quad 0<r<1 .
$$

CONJECTURE 4.2. - We conjecture that for suitably chosen families of $N$-tuples $Z_{N}$ on $S$, where $N \rightarrow \infty$, the associated differences (4.1) become as small as $\mathcal{O}\left(e^{-c N^{1 / 2}}\right)$ outside any given neighborhood of $S$ (with $c>0$ again depending on the neighborhood).

The following conditional result describes candidate $N$-tuples $Z_{N}$ for the conjecture.

Theorem 4.3 (cf. Korevaar [13]). - Let the $N$-tuples $Z_{N}=\left(\zeta_{1}\right.$, $\left.\ldots, \zeta_{N}\right)$, with $\zeta_{k}=\zeta_{k}^{(N)}, N \geq 2$ be Fekete $N$-tuples on $S$ for the potential $\Phi(r)=1 / \sqrt{r^{2}+a^{2}}$ where $a>0$ is arbitrary. In other words, for each $N$, the $N$-tuple of points $\zeta_{1}, \ldots, \zeta_{N}$ minimizes the corresponding function

$$
\sum_{j, k=1}^{N} \frac{1}{\sqrt{\left|x_{j}-x_{k}\right|^{2}+a^{2}}}, \quad x_{1}, \ldots, x_{N} \in S .
$$


If it is true [as we conjecture] that the points in the minimizing $N$-tuples are well-separated, that is, if

$$
\left|\zeta_{j}^{(N)}-\zeta_{k}^{(N)}\right| \geq \frac{\delta}{\sqrt{N}}, \quad j \neq k
$$

for some constant $\delta>0$ independent of $N$, then the corresponding differences (4.1) are of order $\mathcal{O}\left(e^{-c N^{1 / 2}}\right)$ at distance $\geq \epsilon>0$ from $S$ as $N \rightarrow \infty$.

The proof is quite technical. It makes essential use of the fact that a bounded analytic function of two complex variables with many zeros is small provided the zeros are well-separated. If an analytic function of one complex variable on the unit disc is bounded by 1 and has $N$ zeros on a concentric disc of radius $r_{0}<1$, the function will be $\mathcal{O}\left(e^{-c N}\right)$ on the latter disc. For a related result in $q \geq 2$ complex variables one needs separated zeros and then the order of smallness is $\mathcal{O}\left(e^{-c N^{1 / q}}\right)$, cf. [13].

\section{Chebyshev-type quadrature on $S$}

The existence of special $N$-tuples $Z_{N}$ on $S$ with very small differences (4.1) is important for Chebyshev-type quadrature on the sphere.

Relative to normalized area measure $\sigma=\lambda / 4 \pi$, the Chebyshev-type quadrature formula for $S$ with nodes at the points $\zeta_{1}, \ldots, \zeta_{N}$ of $Z_{N}$ has the form

$$
\int_{S} f(\zeta) \mathrm{d} \sigma(\zeta) \approx \frac{1}{N} \sum_{k=1}^{N} f\left(\zeta_{k}\right)
$$

The difference between the two members is the quadrature remainder,

$$
R\left(f, Z_{N}\right)=\int_{S} f(\zeta) \mathrm{d} \sigma(\zeta)-\frac{1}{N} \sum_{k=1}^{N} f\left(\zeta_{k}\right)
$$

The quadrature formula (4.5) is called (polynomially) exact to degree $p$ if $R\left(f, Z_{N}\right)=0$ for all polynomials $f(x)=f\left(x_{1}, x_{2}, x_{3}\right)$ of degree $\leq p$.

We will call $Z_{N}=\left(\zeta_{1}, \ldots, \zeta_{N}\right)$ a "good" $N$-tuple of nodes if formula (4.5) is exact to relatively high degree $p=p_{N}$, or if at least the remainder (4.6) is very small for such polynomials. 
Observe that $U^{\omega}(x)-U\left(x, Z_{N}\right)$ can be interpreted as a quadrature remainder. For $|x|<1$,

$$
U^{\omega}(x)-U\left(x, Z_{N}\right)=\int_{S} \frac{1}{|x-\zeta|} \mathrm{d} \sigma(\zeta)-\frac{1}{N} \sum_{k=1}^{N} \frac{1}{\left|x-\zeta_{k}\right|}=1-U\left(x, Z_{N}\right) .
$$

This fact was used by Korevaar and Meyers [16], cf. Korevaar [13], to derive the following

\section{Equivalence principle}

$Z_{N}$ forms a good $N$-tuple of nodes for Chebyshev-type quadrature on $S$ if and only if the corresponding distribution $\mu\left(Z_{N}\right)$ of charges $1 / N$ gives a nearly constant potential $U\left(\cdot, Z_{N}\right)$ or a small electrostatic field $\mathcal{E}\left(\cdot, Z_{N}\right)$ on a ball (or on all balls) $B(0, r)$ with $0<r<1$. (The differences (4.1) will then also be small for $|x|>R>1$.)

Some precise forms are the following.

Theorem 4.4.- The Chebyshev-type quadrature formula (4.5) for $S$ corresponding to the $N$-tuple $Z_{N}$ is polynomially exact to degree $p$ if and only if

$$
\mathcal{E}\left(x, Z_{N}\right)=\mathcal{O}\left(|x|^{p}\right) \quad \text { for } 0 \leq|x| \leq c<1 .
$$

THEOREM 4.5. - The following two statements, involving a constant $\alpha>0$ and a family of $N$-tuples $Z_{N}$ on $S$ with $N \rightarrow \infty$, are equivalent:

(i) For some (or every) $r \in(0,1)$ there are positive constants $c_{1}(r)$ and $c_{2}(r)$ such that

$$
\sup _{|x| \leq r}\left|\mathcal{E}\left(x, Z_{N}\right)\right| \leq c_{1}(r) \exp \left(-c_{2}(r) N^{\alpha}\right) \quad(N \rightarrow \infty) .
$$

(ii) There are positive constants $c_{3}, c_{4}, c_{5}$ such that

$$
\left|R\left(f, Z_{N}\right)\right| \leq c_{3} \exp \left(-c_{4} N^{\alpha}\right) \sup _{S}|f|
$$

for all polynomials $f$ of degree $\leq c_{5} N^{\alpha}$.

$N$-tuples $Z_{N}$ for which formula (4.5) is exact to degree $p \approx c N^{1 / 3}$ have been described by Korevaar and Meyers in [16], cf. Korevaar [13]. Starting point was the following existence theorem of S. N. Bernstein [1]. 
Proposition 4.6. - For odd $p=2 q-1 \geq 1$, let $M$ be even and

$$
\geq N_{1}(p)=2[2 \sqrt{2}(q+1)(q+4)+1]
$$

where $[\cdot]$ denotes integral part. Then there exist points $z_{1}>z_{2}>\ldots>z_{p}$, $z_{2 q-j}=-z_{j}$ and positive integral weights $m_{j}=m_{2 q-j}, \sum_{j=1}^{p} m_{j}=M$ such that for all polynomials $g(z)$ of degree $\leq p$,

$$
\int_{-1}^{1} g(z) \frac{1}{2} \mathrm{~d} z=\frac{1}{M} \sum_{j=1}^{p} m_{j} g\left(z_{j}\right)
$$

On the sphere, it is now convenient to use coordinates $x, y, z$ as follows:

$$
x=\sin \theta \cos \phi, y=\sin \theta \sin \phi, z=\cos \theta, \quad 0 \leq \theta \leq \pi, 0 \leq \phi<2 \pi,
$$

with normalized area element

$$
\mathrm{d} \sigma(x, y, z)=\frac{1}{4 \pi} \sin \theta \mathrm{d} \theta \mathrm{d} \phi=\frac{1}{4 \pi}|\mathrm{d} z| \mathrm{d} \phi .
$$

Corollary 4.T. - For $p=2 q-1 \geq 1$ we set $N(p)=(p+1) N_{1}(p)$ with $N_{1}(p)$ as above. Let $z_{1}>z_{2}>\ldots>z_{p}$ and the positive integers $m_{1}, \ldots, m_{p}$ also be as in Proposition 4.6 and set

$$
\phi_{j, k}=(k-1) \frac{2 \pi}{(p+1) m_{j}}, \quad j=1, \ldots, p, k=1, \ldots,(p+1) m_{j} .
$$

Then for all polynomials $f(x, y, z) \simeq F(z, \phi)$ of degree $\leq p$.

$$
\int_{S} f \mathrm{~d} \sigma=\frac{1}{4 \pi} \int_{-1}^{1} \int_{0}^{2 \pi} F(z, \phi) \mathrm{d} z \mathrm{~d} \phi=\frac{1}{N(p)} \sum_{j=1}^{p} \sum_{k=1}^{(p+1) m_{j}} F\left(z_{j}, \phi_{j, k}\right) .
$$

In other words, the Chebyshev-type quadrature formula for $S$ with the $N=N(p) \approx \sqrt{2} p^{3}$ distinct nodes

$$
\zeta_{j, k} \simeq\left(z_{j}, \phi_{j, k}\right), \quad 1 \leq j \leq p, 1 \leq k \leq(p+1) m_{j}
$$

is exact to degree $p \approx c N^{1 / 3}$. It follows that parts (ii) and (i) in Theorem 4.5 are true with $\alpha=1 / 3$. 
Theorem 4.3 describes $N$-tuples $Z_{N}$ for which the two parts of Theorem 4.5 will hold with $\alpha=1 / 2$ provided the conjectured separation condition (4.4) is satisfied.

We end with a strong conjecture about Chebyshev-type quadrature on $S$.

Conjecture 4.8 (Korevaar and Meyers [16]). - There exist a constant $c>0$ and special $N$-tuples $Z_{N}$ of distinct nodes, $N \rightarrow \infty$ for which formula (4.5) for $S$ is polynomially exact to degree $p_{N} \approx c \sqrt{N}$. Such $N$-tuples $Z_{N}$ on $S$ are sometimes called spherical $p_{N}$-designs. Using that terminology, our conjecture asserts that for $p \rightarrow \infty$, there exist spherical $p$-designs consisting of $\mathcal{O}\left(p^{2}\right)$ points.

\section{Technical results: comparing energies and potentials}

Let $K$ be any compact set in $\mathbb{R}^{s}$ of positive capacity. The weak ${ }^{*}$ convergence $\omega_{N} \rightarrow \omega$ may be derived from the following comparison of energies:

$$
0<(1-1 / N) I(\omega)-I^{*}\left(\omega_{N}\right) \rightarrow 0
$$

cf. Frostman [5].

For the proof of Theorem 3.1 we need an explicit upper bound in (5.1). The basic ingredients used in the derivation below may be found in the classical work of Pólya and Szegő [22], cf. the author's report [12]. For $\rho>0$ we let $K_{\rho}$ denote the closed $\rho$-neighborhood of $K$.

Proposition 5.1. - In terms of Robin constants $V(\cdot)$ one has for $N \geq 2$ and all $\rho>0$,

$$
0<\left(1-\frac{1}{N}\right) I(\omega)-I^{*}\left(\omega_{N}\right)<\left(1-\frac{1}{N}\right) V(K)-V\left(K_{\rho}\right)+\frac{1}{N} \Phi(\rho) .
$$

\section{Proof}

(i) The first inequality. By the definition of $\omega_{N}$,

$$
I^{*}\left(\omega_{N}\right) \leq \frac{1}{N^{2}} \sum_{\substack{j, k=1 \\ j \neq k}}^{N} \Phi\left(\left|x_{j}-x_{k}\right|\right), \quad \forall x_{1}, \ldots, x_{N} \in K .
$$


Hence by $N^{r}$-fold integration, recalling that $\omega\left(K^{*}\right)=1$,

$$
\begin{aligned}
I^{*}\left(\omega_{N}\right) & =\int_{K \times \cdots \times K^{K}} I^{*}\left(\omega_{N}\right) \mathrm{d} \omega\left(x_{1}\right) \cdots \mathrm{d} \omega\left(x_{N}\right) \\
& <\frac{1}{N^{2}} \sum_{\substack{j, k=1 \\
j \neq k}}^{N} \int_{K^{\prime} \times K} \Phi\left(\left|x_{j}-x_{k}\right|\right) \mathrm{d} \omega\left(x_{j}\right) \mathrm{d} \omega\left(x_{k}\right) \cdot \omega(K)^{N-2} \\
& =\frac{N-1}{N} I(\omega) .
\end{aligned}
$$

(ii) The second inequality. Let $\omega_{N}^{\rho}$ be the probability measure on $K_{\rho}$, obtained from $\omega_{N}$ on $K$ by distributing the charges $1 / N$ at the Fekete points $f_{N k}$ uniformly over the spheres $S\left(f_{N k}, \rho\right)$ of radius $\rho$, centered at those points. The potential $U_{N k}$ of the resulting measure $\omega_{N k}^{\rho}$ on $S\left(f_{N k}, \rho\right)$ satisfies the relations

$$
U_{N k}(x)=\frac{1}{N} \min \left\{\Phi(\rho), \Phi\left(\left|x-f_{N k}\right|\right)\right\} \leq \frac{1}{N^{N}} \Phi\left(\left|x-f_{N k}\right|\right) .
$$

Thus for $j \neq k$,

$$
\begin{aligned}
\int U_{N j} \mathrm{~d} \omega_{N k}^{\rho} & \leq \frac{1}{N^{\top}} \int \Phi\left(\left|x-f_{N j}\right|\right) \mathrm{d} \omega_{N k}^{\rho}(x)=\frac{1}{N} U_{N k}\left(f_{N j}\right) \\
& \leq \frac{1}{N^{2}} \Phi\left(\left|f_{N j}-f_{N k}\right|\right),
\end{aligned}
$$

while for $j=k$,

$$
\int L_{N k} \mathrm{~d} \omega_{N k}^{\rho}=\frac{1}{N^{2}} \Phi(\rho)
$$

Adding up all the terms $\int U_{N j} \mathrm{~d} \omega_{N k}^{\rho}$, it follows that

$$
I\left(\omega_{N}^{\rho}\right) \leq \frac{1}{N^{2}} \sum_{\substack{j, k=1 \\ j \neq k}}^{N} \Phi\left(\left|f_{N j}-f_{N k}\right|\right)+\frac{1}{N} \Phi(\rho)=I^{*}\left(\omega_{N}\right)+\frac{1}{N} \Phi(\rho) .
$$

Finally observing that $I\left(\omega_{N}^{\rho}\right)>V\left(K_{\rho}\right)$, one obtains (5.2).

The minimum property of the Fekete $N$-tuples may be used to compare $U^{\omega_{N}}$ on $K$ with $I^{*}\left(\omega_{N}\right)$, cf. Sjögren [28]: 
Proposition 5.2.- One has

$$
\left(1-\frac{1}{N}\right) U^{\omega_{N}}(x) \geq I^{*}\left(\omega_{N}\right), \quad \forall x \in K
$$

Indeed, for $x \in K$ and each $j$, by the minimum property mentioned above,

$$
\sum_{k=1, k \neq j}^{N} \Phi\left(\left|x-f_{N k}\right|\right) \geq \sum_{k=1, k \neq j}^{N} \Phi\left(\left|f_{N j}-f_{N k}\right|\right) .
$$

Summing over $j=1, \ldots, N$ one finds that $N(N-1) U^{\omega_{N}}(x) \geq N^{2} I^{*}\left(\omega_{N}\right)$.

Combination with Proposition 5.1 gives

Proposition 5.3.- One has

$$
\begin{aligned}
\delta\left(\omega_{N}\right) & \stackrel{\text { def }}{=} \sup _{x \in K^{\prime}}\left\{U^{\omega}(x)-U^{\omega_{N}}(x)\right\} \\
& <V\left(K^{\prime}\right)-\frac{1}{N-1}\left\{N V\left(K_{\rho}\right)-\Phi(\rho)\right\}, \quad \forall \rho>0, N \geq 2 .
\end{aligned}
$$

(Recall for the proof that $U^{\omega}(x) \leq V(K)=I(\omega)$ on $K$.)

We wish to minimize the final member of (5.7) in the case where $K$ is the closure of a well-behaved domain $\Omega$. What we need here is

Proposition 5.4 (cf. Korevaar and Monterie [17], Monterie [21]). - If $\Omega$ is convex or $\Sigma=\partial \Omega$ is of class $C^{1 . \alpha}$ and $K=\operatorname{clos} \Omega$, there are positive constants $A$ and $B$ such that

$$
\operatorname{cap} K_{\rho} \leq \operatorname{cap} K+A \rho, \quad V\left(K_{\rho}\right) \geq V(K)-B \rho, \quad \forall \rho>0 .
$$

Proof. - In the convex case we may take the origin at the center of a maximal ball in $\Omega$. Denoting the ball by $B(0, r)$, one may use geometric arguments to show that $K_{\rho}$ is contained in the set $(1+\rho / r) K$, hence cap $K_{\rho} \leq(1+\rho / r) \operatorname{cap} K$.

In the $C^{1, \alpha}$ case one may use the level surfaces of the Green's function $G=G(y, \infty)$ for $K^{\infty}$ with pole at $\infty$. These must be "well-separated" since $|\operatorname{grad} G|$ will be bounded by a constant $C$ on $K^{\infty}$, cf. Widman ([35], [36]). In particular $K_{\rho}$ will (for small $\rho$ ) be enclosed by the level 
surface $\Sigma_{\delta}:\{G=\delta\}$ with $\delta=C \rho$. Now for $s \geq 3$, the exterior of $\Sigma_{\delta}$ will have Green's function with pole at $\infty$ equal to $(G-\delta) /(1-\delta)$, hence by $(2.6),\left(\operatorname{cap} \Sigma_{\delta}\right)^{s-2}=(\operatorname{cap} K)^{s-2} /(1-\delta)$. Conclusion: $\operatorname{cap} K_{\rho} \leq$ $(\operatorname{cap} K) /(1-C \rho)^{1 /(s-2)}$. For $s=2$, one finds that $V\left(K_{\rho}\right) \geq V(K)-C \rho$.

To complete the proof one uses the relation between the capacity and the Robin constant, cf. (2.5).

Combining Propositions 5.3 and 5.4 we can prove the crucial estimate (3.6) required for the proof of Theorem 3.1:

Proposition 5.5 (cf. Korevaar and Monterie [17]). - Under the conditions on $\Omega$ given in Theorem 3.1 or Proposition 5.4 , there is a constant $c$ depending only on $\Omega$ such that

$$
\delta\left(\omega_{N}\right) \leq\left\{\begin{array}{ll}
\frac{c}{N^{1 /(s-1)}} & \text { if } s \geq 3, \\
\frac{c \log N}{N} & \text { if } s=2 .
\end{array} \quad(N \geq 2) .\right.
$$

The proof is by minimization of the final member in (5.7) with respect to $\rho$, after one has replaced $V\left(K_{\rho}\right)$ by its minorant $V(K)-B \rho$ from (5.8).

\section{Additional technical results: integral representations and inequalities}

The convex or $C^{1, \alpha}$ domains $\Omega$ of Theorem 3.1 are special cases of Lipschitz domains: bounded domains $\Omega$ with Lipschitz boundary $\Sigma$. For such domains Dahlberg has proved an important result on harmonic measure. To simplify the corresponding representations, we divide the Lebesgue measure $\lambda$ on the boundary surface $\Sigma$ by the area $\lambda(S)$ of the unit sphere $S \subset \mathbb{R}^{s}$. The adjusted area measure will be $\sigma(y)=\lambda(y) / \lambda(S)$.

Theorem 6.1 (Dahlberg [2], theorem 3). - Let $\Omega$ be a Lipschitz domain and let $G(y)=G(y, x)$ be the Green's function for $\Omega$ with pole at $x$. Then the inward unit normal $n_{y}$ and the corresponding normal derivative

$$
\begin{gathered}
\frac{\partial G}{\partial n_{y}}(y)=\lim _{\epsilon \downarrow 0} G\left(y+\epsilon n_{y}\right) \\
-72-
\end{gathered}
$$


exist for almost all $y \in \Sigma=\partial \Omega$ relative to $\sigma$. The function $\partial G / \partial n_{y}$ is positive a.e. and integrable over $\Sigma$. For the measurable subsets $E \subset \Sigma$, the harmonic measure

$$
\omega_{x}(E) \text { equals } \int_{E} \frac{\partial G}{\partial n_{y}}(y, x) \mathrm{d} \sigma(y) .
$$

Not only is $\omega_{x}$ absolutely continuous with respect to $\sigma$, but the converse is also true.

By the definition of harmonic measure, Theorem 6.1 implies a representation formula for harmonic functions which are continuous up to the boundary. In the case of convex or $C^{1, \alpha}$ domains this formula can be extended to potentials of measures on the boundary by a limit process involving monotone or dominated convergence. The result is given in Theorem 6.2.

There are corresponding results for the exterior domain $\Omega^{\infty}$ of $\Sigma$. In the special case of the Green's function $G(y, \infty)$ with pole at $\infty$ one will thus obtain a representation for $L(u)=\lim |z|^{s-2} u(z)$ at $\propto$. Observe that normals must always point into the domain considered, hence for the exterior domain one uses the outward normal to $\Sigma$.

Theorem 6.2 (cf. Korevaar and Monterie [17]). - For convex or $C^{1, \alpha}$ domains $\Omega$ as in Theorem 3.1 and for harmonic functions $u$ on $\Omega$ or $\Omega^{\infty}$ that are continuous up to the boundary $\Sigma$, and also for potentials $u=U^{\rho}$ with $\operatorname{supp} \rho \subset \Sigma$,

$$
\begin{gathered}
u(x)=\int_{\Sigma} u(y) \frac{\partial G}{\partial n_{y}}(y, x) \mathrm{d} \sigma(y), \quad x \in \Omega, \\
L(u) \stackrel{\text { def }}{=} \lim _{z \rightarrow \infty}|z|^{s-2} u(z)=\int_{\Sigma} u(y) \frac{\partial G}{\partial n_{y}}(y, \infty) \mathrm{d} \sigma(y) .
\end{gathered}
$$

As a by-product of our proof for the second formula (6.2) we have obtained the following representation for the equilibrium measure.

Theorem 6.3 (cf. Korevaar and Monterie [17]). - If $K$ is the closure of a Lipschitz domain $\Omega$, the equilibrium measure $\omega$ for $K$ on $\Sigma=\partial \Omega$ has the representation

$$
\mathrm{d} \omega(y)= \begin{cases}V \frac{\partial G}{\partial n_{y}}(y, \infty) \mathrm{d} \sigma(y) & \text { if } s \geq 3, \\ \frac{\partial G}{\partial n_{y}}(y, \infty) \mathrm{d} \sigma(y) & \text { if } s=2 .\end{cases}
$$


For the application of Theorem 6.2 in the proof of Theorem 3.1 we need bounds on the normal derivatives of the Green's functions. Let us first suppose that $\Sigma=\partial \Omega$ is of class $C^{1, \alpha}$ and that $x \in \Omega$. Then the following results are known from the work of Lyapunov, Eydus and Widman, cf. Widman ([35], [36]) who actually considered somewhat more general Lyapunov-Dini domains $\Omega$. The normal derivative $\left(\partial G / \partial n_{y}\right)(y, x)$ is continuous on $\Sigma$ and strictly positive, and

$$
\frac{\partial G}{\partial n_{y}}(y, x) \leq \frac{c}{|x-y|^{s-1}} \leq \frac{c}{d_{x}^{s-1}}, \forall y \in \Sigma .
$$

(As before, $d_{x}=d(x, \Sigma)$.) One may use Kelvin inversion to obtain related results for exterior Green's functions $G(y, x)$. In particular the derivative $\left(\partial G / \partial n_{y}\right)(y, \infty)$ will be continuous on $\Sigma$ and strictly positive.

Next let $\Sigma$, or more precisely $\Omega$, be convex. In this case the level surfaces for the interior Green's functions $G(y, x)$ and for the exterior Green's function $G(y, \infty)$ are also convex. This was proved by Gabriel ([6], [7]) for $s=3$ but his proof readily extends to other dimensions (the case $s=2$ is classical). (There is an extensive literature on convexity of level sets, cf. Kawohl [9] and N. J. Korevaar [18] for references.) As a corollary one may deduce that the normal derivative $\left(\partial G / \partial n_{y}\right)(y, x)$ is bounded above on $\Sigma$ by a constant $A_{x} \leq M\left(d_{x}\right)$ and that the derivative $\left(\partial G / \partial n_{y}\right)(y, \infty)$ is bounded from below by a positive constant $m(\infty)$, cf. Korevaar and Monterie [17]. Comparison of $\Omega$ with a half-space readily shows that one may take $M\left(d_{x}\right)=2(s-2) / d_{x}^{s-1}$ if $s \geq 3, M\left(d_{x}\right)=2 / d_{x}$ if $s=2$.

\section{References}

[1] Bernstein (S. N.) .- On quadrature formulas with positive coefficients, Izv. Akad. Nauk SSSR (Russian), Ser. Mat. 1, nº 4 (1937), pp. 479-503; Collected Works, vol. II. Izdat. Akad. Nauk SSSR. Moscow, 1954, pp. 205-227; See also the announcements 'Sur les formules de quadrature à coefficients non négatifs et abscisses équidistantes' and 'Modifications de la formule de quadrature de Tchebycheff' in C.R. Acad. Sci. Paris 204 (1937), pp. 1294-1296 and 1526-1529.

[2] Dahlberg (B. E. J.) .- Estimates of harmonic measure, Arch. Rat. Mech. Anal. 65 (1977), pp. 275-288.

[3] Dahlberg (B. E. J.) .- On the distribution of Fekete points, Duke Math. J. 45 (1978), pp. 537-542.

[4] FeKete (M.) .- Über die Verteilung der Wurzeln bei gewissen algebraischen Gleichungen mit ganzzahligen Koeffizienten, Math. Z. 17 (1923), pp. 228-249. 
[5] Frostman (O.) .- Potentiel d'équilibre et capacité des ensembles avec quelques applications à la théorie des fonctions, Dissertation, Lunds Univ. Mat. Sem. 3 (1935), pp. 1-118.

[6] Gabriel (R. M.) - - An extended principle of the maximum for harmonic functions in 3 dimensions, J. London Math. Soc. 30 (1955), pp. 388-401.

[7] Gabriel (R. M.) .- A result concerning convex level surfaces of 3-dimensional harmonic functions, J. London Math. Soc. 32 (1957), pp. 286-294.

[8] Gilbarg (D.) and TRudinger (N.S.) .- Elliptic partial differential equations of second order, Grundl. d. math. Wiss. 224, Springer-Verlag, New York, 1977.

[9] KAWOHL (B.) .- Rearrangements and convexity of level sets in PDE, Lecture Notes in Math. 1150, Springer, Berlin, 1985.

[10] KorevaAr (J.) . - Asymptotically neutral distributions of electrons and polynomial approximation, Ann. of Math. 80 (1964), pp. 403-410.

[11] KorevaAr (J.) .- Equilibrium distributions of electrons on roundish plane conductors I, II, Indag. Math. 36 (1974), pp. 423-456.

[12] KorevaAr (J.) . - Problems of equilibrium points on the sphere and electrostatic fields, Univ. of Amsterdam, Math. Dept. Report 1976-03.

[13] KorevaAR (J.) .- Chebyshev-type quadratures: use of complex analysis and potential theory, in: Complex potential theory (P. M. Gauthier and G. Sabidussi, eds.), Kluwer, Dordrecht, 1994, pp. 325-364.

[14] KorevaAr (J.) and Geveci (T.) . - Fields due to electrons on an analytic curve, SIAM J. Math. Anal. 2 (1971), pp. 445-453.

[15] Korevaar (J.) and Kortram ( R. A.) .- Equilibrium distributions of electrons on smooth plane conductors, Indag. Math. 45 (1983), pp. 203-219.

[16] KorevaAr (J.) and Meyers (J. L. H.) .- Spherical Faraday cage for the case of equal point charges and Chebyshev-type quadrature on the sphere, Integral Transforms and Special Functions 1 (1993), pp. 105-117.

[17] Korevaar (J.) and Monterie ( M. A.) .- Electrostatic fields due to distributions of equal point charges on convex conductors in $\mathbb{R}^{3}$ with minimal energy. Math. Dept., Univ. of Amsterdam, Preprint, 1995.

[18] Korevaar (N. J.) .- Convexity of level sets for solutions to elliptic ring problems, Comm. Partial Differential Equations 15 (1990), pp. 541-556.

[19] KöVARI (T.) .- On the distribution of Fekete points II, Mathematika 18 (1971), pp. 40-49.

[20] Kövari (T.) and Pommerenke (C.) .- On the distribution of Fekete points, Mathematika 15 (1968), pp. 70-75.

[21] Monterie (M. A.) .- - Studies in potential theory, Ph. D. thesis, Free University, Amsterdam, 1995.

[22] Pólya (G.) and Szegö (G.) .- Über den transfiniten Durchmesser (Kapazitätskonstante) von ebenen und räumlichen Punktmengen, J. reine und angew. Math. 165 (1931), pp. 4-49.

[23] Pommerenke (C.) .- Polynome und konforme Abbildung, Monatsh. Math. 69 (1965), pp. 58-61.

[24] Pommerenke (C.) .- - Über die Verteilung der Fekete-Punkte, Math. Ann. 168 (1967), pp. 111-127. 
[25] Pommerenke (C.) - - Über die Verteilung der Fekete-Punkte II, Math. Ann. 179 (1969), pp. 212-218.

[26] Robin (G.) .- Sur la distribution de l'électricité à la surface des conducteurs fermés et des conducteurs ouverts, Ann. École Norm. Sup. (3) 3 (1886), Supplément.

[27] SCHUR (I.) .- Über die Verteilung der Wurzeln bei gewissen algebraischen Gleichungen mit ganzzahligen Koeffizienten, Math. Z. 1 (1918), pp. 377-402.

[28] SJÖGREN (P.) .- On the regularity of the distribution of the Fekete points of a compact surface in $R^{n}$, Ark. Mat. 11 (1973), pp. 147-151.

[29] Stieltjes (T. J.) . - Sur certains polynômes qui vérifient une équation différentielle linéaire du second ordre et sur la théorie des fonctions de Lamé, Acta Math. 6 (1885), pp. 321-326; Euvres Complètes, vol. I. Noordhoff, Groningen, 1914, pp. 434-439; CEuvres Complètes - Collected Papers, vol. I (enlarged edition, G. van Dijk, ed.); Springer, Berlin, 1993, pp. 522-527.

[30] Stieltues (T. J.) .- Sur quelques théorèmes d'algèbre, C.R. Acad. Sci. Paris 100 (1885), pp. 439-440; Euvres Complètes, vol. I, pp. 440-441; Euvres Complètes - Collected Papers, vol. I, pp. 528-529.

[31] Stieltjes (T. J.) .- Sur les polynômes de Jacobi, C.R. Acad. Sci. Paris 100 (1885), pp. 620-622; CEuvres Complètes, vol. I, pp. 442-444; Euvres Complètes - Collected Papers, vol. I, pp. 530-532.

[32] Stieltjes (T. J.) . - Sur les racines de l'équation $X_{n}=0$, Acta Math. 9 (1886), pp. 385-400 ; Euvres Complètes, vol. II. Noordhoff, Groningen, 1918, pp. 73-88; Euvres Complètes - Collected Papers, vol. II (enlarged edition, G. van Dijk. ed.); Springer, Berlin, 1993, pp. 77-92.

[33] Szegö (G.) .- Orthogonal polynomials, Amer. Math. Soc. Colloq. Publ. 23: Providence, R.I., Fourth edition, 1975.

[34] VAN Assche (W.) .- The impact of Stieltjes' work on continued fractions and orthogonal polynomials, In: T. J. Stieltjes, Euvres Complètes - Collected Papers. vol. I (enlarged edition, G. van Dijk, ed.). Springer. Berlin, 1993, pp. 5-37.

[35] Widman (K.-O.) .- On the boundary values of harmonic functions in $\mathbb{R}^{3}$, Arkiv för Mat. 5, nº 14 (1964), pp. 221-230.

[36] WIDMAN (K.-O.) .- Inequalities for the Green function and boundary continuity of the gradient of solutions of elliptic differential equations, Math. Scand. 21 (1967), pp. 17-37. 